\title{
Patient-Customized Oligonucleotide Therapy for a Rare Genetic Disease
}

\author{
J. Kim, C. Hu, C. Moufawad El Achkar, L.E. Black, J. Douville, A. Larson, \\ M.K. Pendergast, S.F. Goldkind, E.A. Lee, A. Kuniholm, A. Soucy, J. Vaze, \\ N.R. Belur, K. Fredriksen, I. Stojkovska, A. Tsytsykova, M. Armant, R.L. DiDonato, \\ J. Choi, L. Cornelissen, L.M. Pereira, E.F. Augustine, C.A. Genetti, K. Dies, \\ B. Barton, L. Williams, B.D. Goodlett, B.L. Riley, A. Pasternak, E.R. Berry, \\ K.A. Pflock, S. Chu, C. Reed, K. Tyndall, P.B. Agrawal, A.H. Beggs, P.E. Grant, \\ D.K. Urion, R.O. Snyder, S.E. Waisbren, A. Poduri, P.J. Park, A. Patterson, A. Biffi, \\ J.R. Mazzulli, O. Bodamer, C.B. Berde, and T.W. Yu
}

\section{SUMMARY}

Genome sequencing is often pivotal in the diagnosis of rare diseases, but many of these conditions lack specific treatments. We describe how molecular diagnosis of a rare, fatal neurodegenerative condition led to the rational design, testing, and manufacture of milasen, a splice-modulating antisense oligonucleotide drug tailored to a particular patient. Proof-of-concept experiments in cell lines from the patient served as the basis for launching an "N-of-1" study of milasen within 1 year after first contact with the patient. There were no serious adverse events, and treatment was associated with objective reduction in seizures (determined by electroencephalography and parental reporting). This study offers a possible template for the rapid development of patient-customized treatments. (Funded by Mila's Miracle Foundation and others.)

The authors' full names, academic degrees, and affiliations are listed in the Appendix. Address reprint requests to Dr. Yu at the Division of Genetics and Genomics, Boston Children's Hospital, 300 Longwood Ave., Mailstop BCH3150, Boston, MA 02115, or at timothy.yu@ childrens.harvard.edu.

Drs. Kim and Hu contributed equally to this article.

This article was published on October 9 , 2019, at NEJM.org.

N Engl J Med 2019;381:1644-52. DOI: 10.1056/NEJMoa1813279

Copyright @ 2019 Massachusetts Medical Society.

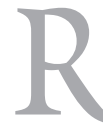

ARE DISEASES IN AGGREGATE AFFECT APPROXIMATELY 30 MILLION PERsons in the United States alone. ${ }^{1}$ Although next-generation sequencing is revolutionizing their diagnosis, the sheer number of distinct conditions (more than 7000 [https://globalgenes.org/rare-list]) and the limited number of patients affected by each rare disease present major challenges for drug development.

This report shows a path to personalized treatment for patients with orphan diseases. It describes the identification of a novel mutation in a child with neuronal ceroid lipofuscinosis 7 (CLN7, a form of Batten's disease), a rare and fatal neurodegenerative disease. ${ }^{2,3}$ Identification of the mutation was followed by the development and clinical deployment, within 1 year, of a tailored drug to treat the patient (Fig. 1A).

\section{CLINICAL PRESENTATION}

A 6-year-old girl presented with the insidious onset of blindness, ataxia, seizures, and developmental regression. The parents' first concerns dated back to when the girl was 3 years of age, when her right foot began to turn inward. When she was 4 years of age, her family noticed her pulling books close to her face at bedtime. At 5 years of age, she came to medical attention because of modest language and social regression, as well as increased clumsiness and stumbling. In the months before she turned 6 years of age, the progression of symptoms accelerated, and she was 


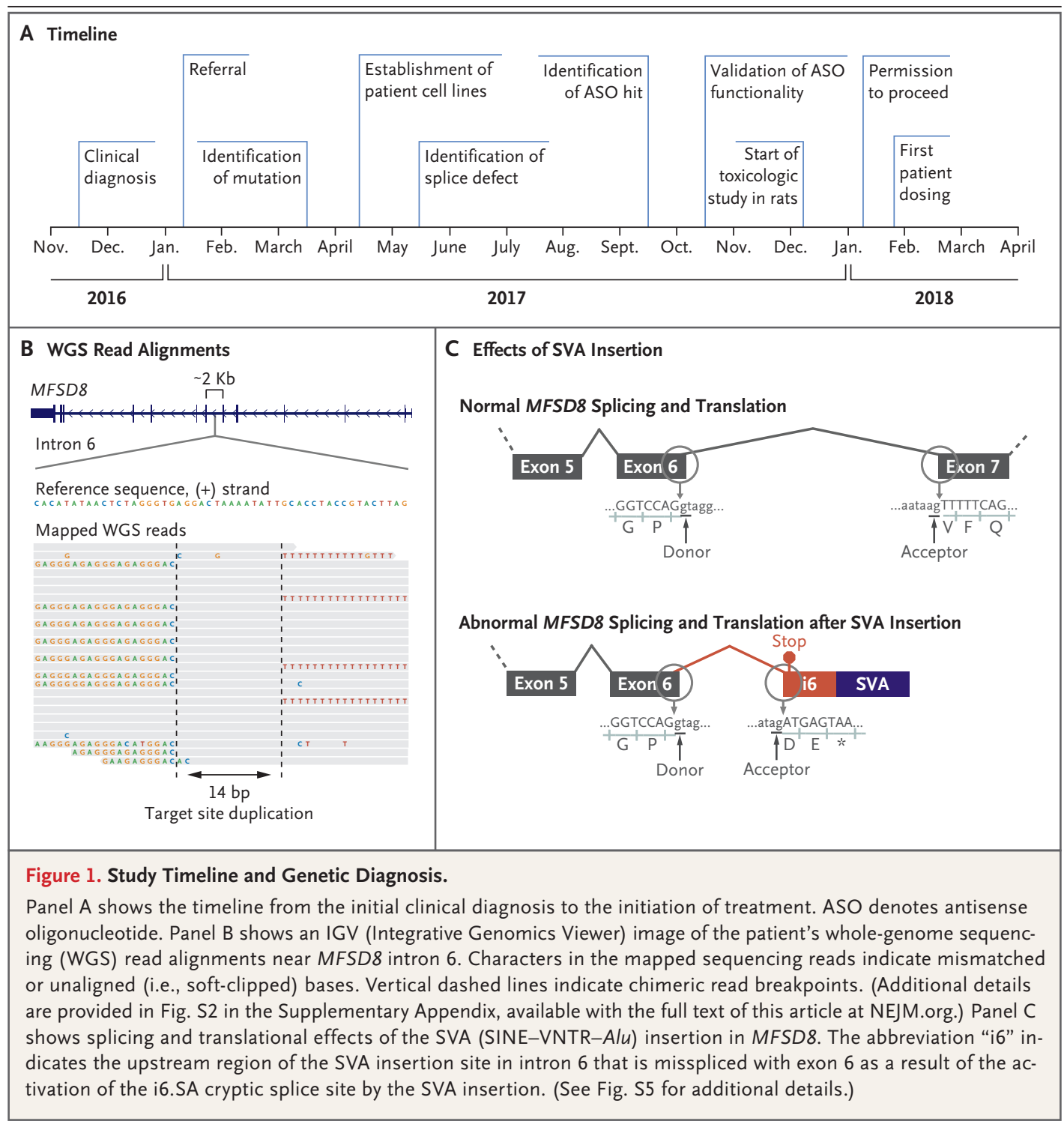

hospitalized after the rapid development of loss of vision, frequent falls, dysarthria, and dysphagia. Magnetic resonance imaging (MRI) of the head revealed mild cerebral and cerebellar atrophy (Fig. S1A), and 24-hour electroencephalography (EEG) revealed several subclinical generalized seizures. An extensive laboratory workup for mitochondrial or metabolic diseases was initially unrevealing, until a skin biopsy was performed. The biopsy showed abnormal, electron-dense, lysosomal inclusions in a swirling fingerprint pattern (Fig. S1B). ${ }^{3}$ This pattern was recognized to be diagnostic of Batten's disease, which is autosomal recessive and affects the retina and the central nervous system (CNS). Genetic panel testing (including deletion-duplication analysis) for known Batten's disease genes revealed heterozygosity for a single known pathogenic mutation (NM_152778.3: c.1102G $\rightarrow$ C; ClinVar accession number, RCV000149775.5) in the gene MFSD8 (also known as CLN7). No second mutation could be found. The family was offered enrollment in a research study for further diagnostic workup.

\section{METHODS}

After obtaining written informed consent from the patient's parents, we obtained blood and skin samples for genomic and molecular characterization under an appropriate human subjects re- 
search protocol approved by the institutional review board of Boston Children's Hospital. A full description of the methods of the subsequent workup is provided in the Supplementary Appendix.

After informed consent was obtained from the patient's parents, treatment was conducted under an expanded-access investigational clinical protocol authorized by the Food and Drug Administration (FDA), with the concurrence of the chair of the Boston Children's Hospital institutional review board. All the authors vouch for the accuracy and completeness of the data and for the fidelity of the study to the protocol, available at NEJM.org.

RESULTS

\section{MUTATION IDENTIFICATION}

Whole-genome sequencing was undertaken to search for the missing second mutation, which was hypothesized to be a noncoding mutation or a structural variant missed by standard clinical sequencing. Careful inspection of sequencing data revealed a cluster of chimeric reads deep in MFSD8 intron 6 (Fig. 1B), detected in both the proband and the mother (Fig. S2A). Chimeric reads in this cluster were fused either to unmapped poly-T sequences on one end or to unmapped hexameric repeat sequences (AGAGGG) on the other end, which suggested the presence of a DNA insertion beginning and ending with these motifs. Chimeric breakpoints were offset by $14 \mathrm{bp}$, suggesting duplication of an endogenous target sequence (Fig. 1B).

We deduced that these features were consistent with the insertion of an SVA (SINE-VNTR-Alu) retrotransposon. ${ }^{4}$ Indeed, amplification by polymerase chain reaction (PCR) across the breakpoints revealed an approximately $2-k b$ insertion in the proband and the mother that was confirmed by RepeatMasker analysis to be an SVA retrotransposon. The segregation of the variant within this family was confirmed in a Clinical Laboratory Improvement Amendments-certified laboratory to match recessive inheritance (the proband is compound heterozygous for the SVA insertion and c.1102G $\rightarrow \mathrm{C}$, and the parents are heterozygous carriers). The SVA insertion was also correctly detected by automated analysis with the
Tea algorithm. ${ }^{5}$ This insertion has not been reported in the literature and does not appear in dbRIP, a database of retrotransposon insertions in the human population. ${ }^{6}$ Its absence was also confirmed in more than 800 persons by Tea analysis of publicly available human whole-genome sequencing data sets. (Additional details of the results of these analyses are provided in Figs. S2 through S4.)

Because SVA insertions have been reported to modulate splicing of nearby genes, ${ }^{7}$ we examined splicing patterns in the patient's family. RNA sequencing (RNA-seq) and reverse-transcriptasePCR analyses revealed missplicing of exon 6 into a cryptic splice-acceptor site (i6.SA) in MFSD8 intron 6, in a location 119 bp upstream from the SVA insertion site, in blood samples and lymphoblasts from the proband and mother (but not the father or an unaffected sibling). This missplicing precisely segregated with the SVA insertion (Fig. 1C and Fig. S5) and was predicted to lead to premature translational termination, which supported the pathogenicity of the insertion.

\section{DEVELOPMENT OF AN ANTISENSE OLIGONUCLEOTIDE DRUG}

Nusinersen is an FDA-approved antisense oligonucleotide drug for spinal muscular atrophy ${ }^{8-10}$ that changes the splicing pattern of the SMN2 RNA. ${ }^{11-13}$ Reasoning that an antisense oligonucleotide might be similarly used to correct missplicing and restore MFSD8 expression in our patient, we designed antisense oligonucleotides to target the i6.SA cryptic splice-acceptor site and nearby splicing enhancers (Fig. 2A). When we tested these antisense oligonucleotides in patient fibroblasts, we identified three that boosted normal:mutant splicing ratios by a factor of 2.5 to 3. TY777 was the most efficacious (Fig. 2B) and became our lead candidate; we dubbed it "milasen."

Milasen is a 22-nucleotide antisense oligonucleotide with the same backbone and sugar chemistry modifications (phosphorothioate and 2'-O-methoxyethyl) as nusinersen. ${ }^{8,13}$ Doseresponse analysis indicated that its half-maximal potency was in the nanomolar range. RNA-seq from patient fibroblasts showed that milasen treatment more than tripled the amount of normal (exon 6-exon 7) splicing (Fig. 2C). Com- 


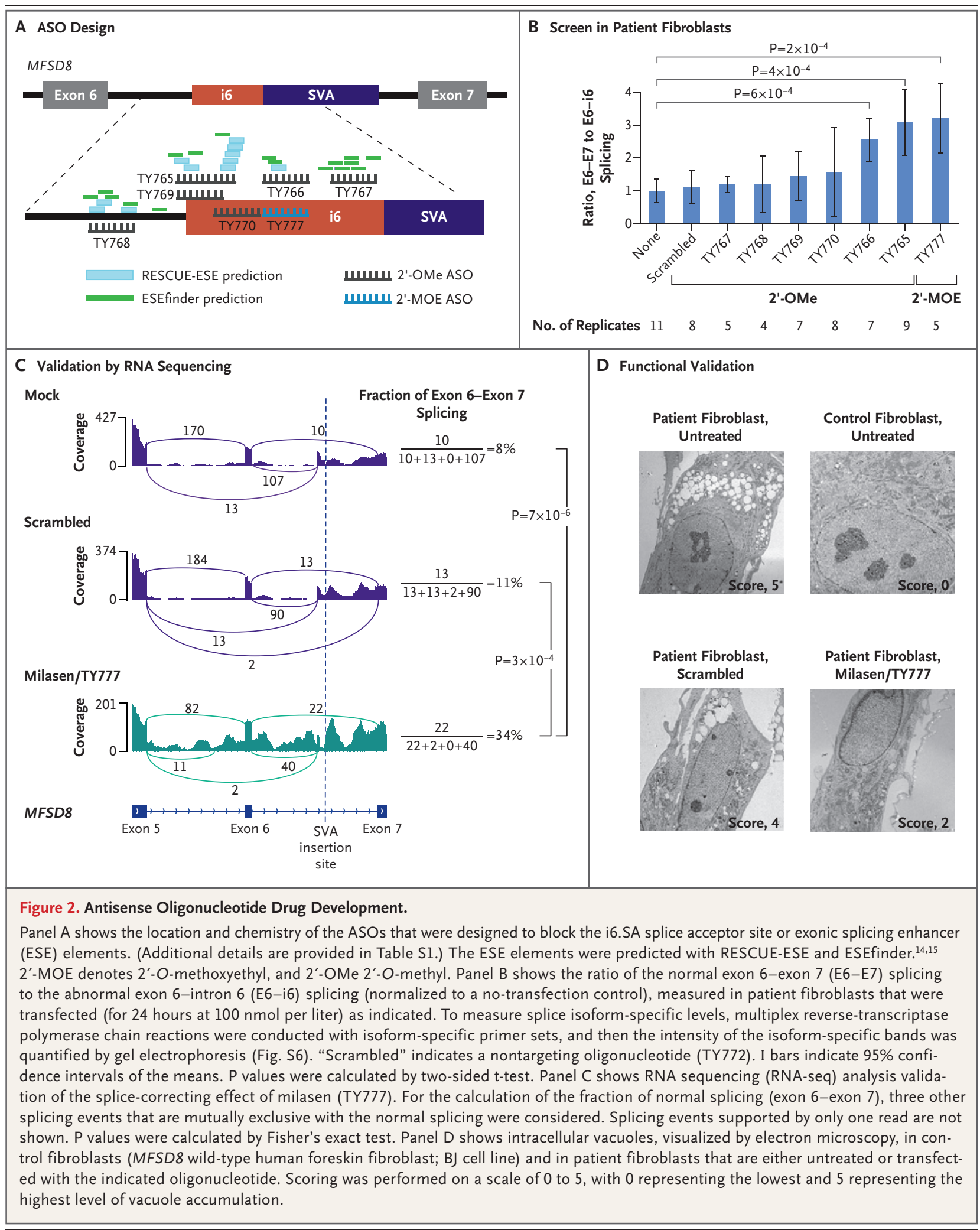


puter-based sequence analysis of milasen showed little potential for off-target binding in the human genome.

Fibroblasts from the patient had several cellular phenotypes characteristic of lysosomal dysfunction, including intracellular vacuolation, ${ }^{16}$ increased total lysosomal mass, ${ }^{17}$ autofluorescence, miscompartmentalization of lysosomal enzyme activity, and reduced autophagic flux ${ }^{18}$ (Fig. 2D). All these phenotypes were alleviated by milasen administration, indicating that treatment could rescue not only splicing but also function. (Additional details regarding the analyses we performed during the development of milasen are provided in Tables S1 and S2 and Figs. S6 through S12.)

STUDIES IN SUPPORT OF AN INVESTIGATIONAL NEW DRUG APPLICATION

The patient's condition continued to deteriorate. Clinical evaluation at 7 years of age revealed an inability to make discernible words, dysphagia (prompting gastrostomy tube placement), the need for substantial support to walk, and 15 to 30 overt seizures per day. She did, however, remain alert and reactive to familiar stimuli and responded happily to hearing her favorite books and songs.

In the largest published case series of CLN7 Batten's disease, the mean age of patients at onset was 3.3 years, and seven patients died at a mean age of 11.5 years. ${ }^{19}$ Considering our patient's clinical prognosis, and after appropriate scientific and ethics review, we filed for FDA permission to initiate clinical investigational treatment under an Expanded Access Investigational New Drug application. Milasen drug substance (18 g) was manufactured and formulated for clinical administration. To identify potential hazards, we administered milasen to rats by intrathecal injection at three doses: $0.06 \mathrm{mg}, 0.25$ $\mathrm{mg}$, and $1.0 \mathrm{mg}$ (approximately 2.5 times, 10 times, and 42 times, respectively, the typical clinical dose of nusinersen, after compartmental scaling considerations). No adverse effects were observed in the group that received $0.06 \mathrm{mg}$. At higher doses, some animals (half of those in the group that received $0.25 \mathrm{mg}$ and most of those in the group that received $1.0 \mathrm{mg}$ ) had hindlimb weakness, which resolved by approximately 24 hours after each dose. (For additional details, including multidose experiments, see Fig. S13 and Supplementary Text.) These results provided translatable indicators of potential toxic effects that could be readily monitored and guided our clinical protocol design.

\section{CLINICAL STUDY}

We initiated a clinical study of milasen in our patient 1 month after we started the toxicology studies in animals (Fig. 1A). Our regimen was roughly modeled after nusinersen, ${ }^{8-10}$ because of the parallels between the two drugs (i.e., they are similarly sized antisense oligonucleotides with identical chemical modifications, targeting the same tissue [the CNS]). Milasen was given by intrathecal bolus injection, starting at $3.5 \mathrm{mg}$ and increasing approximately every 2 weeks up to $42 \mathrm{mg}$ (Fig. 3A and Fig. S14A). A dose of $42 \mathrm{mg}$ was chosen because antisense oligonucleotides administered intrathecally to humans or nonhuman primates distribute to the brain (the target of milasen) with approximately one third the efficiency with which they distribute to the spinal cord (the target of nusinersen), ${ }^{8,13}$ and $42 \mathrm{mg}$ of milasen is the molar equivalent of 3 times the typical nusinersen dose (12 mg). After dose escalation, two additional loading doses were administered, followed by maintenance dosing approximately every 3 months.

\section{CLINICAL OUTCOMES}

Adverse Events, Pharmacokinetics, and Imaging

Through the first year of treatment, no serious adverse events occurred. No clinically significant adverse changes were observed in vital signs; in the results of physical examination, including assessments of strength, gait, and sensory testing; or in clinical laboratory test profiles. Pharmacokinetic analysis indicated a general trend of doseproportional increases in drug levels in cerebrospinal fluid (Fig. 3B) with only limited systemic exposure. MRI of the head showed continued brain volume loss 7 months after treatment initiation, extending a trend that had been observed over the previous 3 years. In a previous study of gene therapy for CLN5 Batten's disease, animals treated after symptom onset continued to have brain volume loss over a period of 20 months, despite treatment slowing the progression of symptoms. ${ }^{20}$ (Details of the results of these analyses in our study are provided in Tables S3 through S5 and Figs. S14 through S18.) 


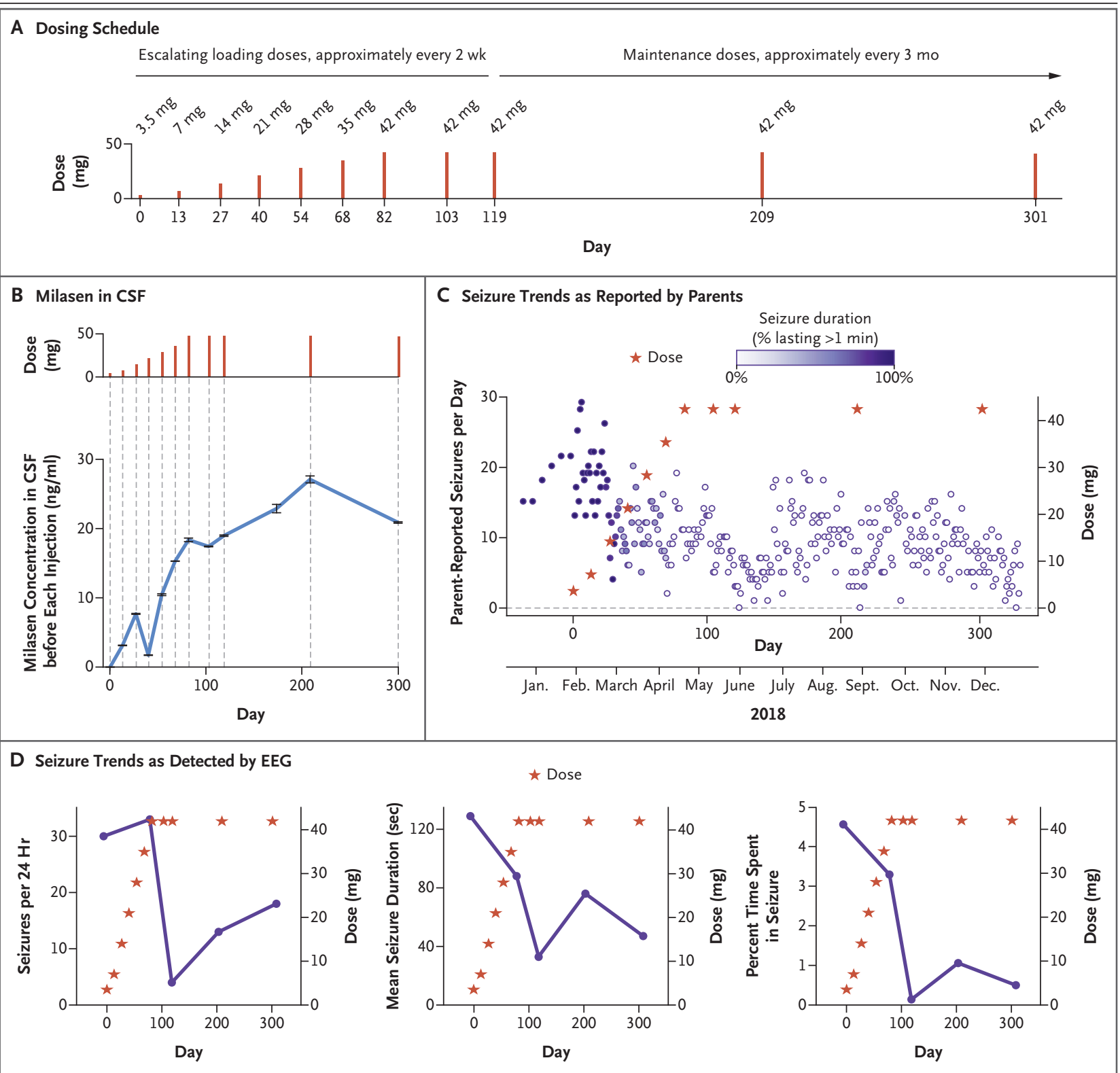

Figure 3. N-of-1 Clinical Study.

Panel A shows the dosing schedule. (Additional details are provided in Fig. S14A.) Panel B shows the concentration of milasen in cerebrospinal fluid (CSF) before each administration (trough). An additional measurement of the concentration in CSF was obtained at day 174 (without concurrent dose administration). I bars indicate the minimum and maximum values of duplicate measurements. Trough levels rose steadily in a dose-proportional fashion until day 40, at which point they dropped to $1.7 \mathrm{ng}$ per milliliter and then resumed their rise with repeated dosing up to a plateau of 18 to $27 \mathrm{ng}$ per milliliter. The dip at day 40 may have been due to a CSF leak, given its coincident timing with a post-lumbar puncture headache after the previous dose. A similar plateauing of CSF trough levels was observed in a previous study of intrathecally delivered nusinersen ( 9 to $11 \mathrm{ng}$ per milliliter after four repeated doses of $12 \mathrm{mg}$ ). ${ }^{8}$ Panel $\mathrm{C}$ shows the trends in seizure frequency and duration as reported in a seizure diary recorded by the parents. Seizures were all of the same type: sudden startle followed by uncontrollable, untriggered laughter that was different from the patient's natural laugh, at times accompanied by an increase in the nonspecific repetitive hand movements she had at baseline. Panel $D$ shows the trends in seizure activity as detected by electroencephalography (EEG). In a comparison of the means of the initial two recordings and the subsequent three recordings, the daily seizure count, seizure duration, and percent cumulative time spent in seizure decreased by $63 \%$ (from 31.5 to 11.7 per day), $52 \%$ (from 108 seconds to 52 seconds), and $85 \%$ (from $3.9 \%$ to $0.6 \%$ ), respectively. 
Neurologic and Neuropsychological Assessments

Testing with the use of Vineland Adaptive Behavior Scales, Second Edition (Vineland-II), showed declines in 7 of 11 neurologic and neuropsychological subscores between day -100 and day -6 . Of the 4 subscores that did not decline, 2 remained the same and 2 improved in the 3 months before the initiation of the clinical study. These declines represented ongoing losses of some of the patient's few remaining adaptive skills in the domains of communication, daily living skills, and socialization. Subscores tended to stabilize in the period from the start of the clinical study to approximately 3 months ( 3 declined, 6 remained the same, and 2 improved from day -6 to day 107) and from 3 months to 6 months (4 declined, 2 remained the same, and 5 improved from day 107 to day 203). The results of testing with the Bayley Scales of Infant and Toddler Development, Third Edition; Global Motor Function Measure-88 (GMFM-88) scores; and results of sensory threshold testing remained stable throughout treatment. (Details of these results are provided in Tables S4 and S5 and Figs. S15 through S17.)

\section{Seizures}

At baseline, our patient was having approximately 15 to 30 seizures per day, each lasting 1 to $2 \mathrm{~min}$ utes (Fig. 3C). Over the course of the clinical study, the frequency decreased to between 0 and 20 seizures per day, and the duration of each seizure decreased to less than 1 minute. These trends were corroborated by overnight electroencephalographic monitoring (Fig. 3D), which indicated that both the frequency and duration of seizures decreased by greater than $50 \%$ (comparing the initial two recordings and the subsequent three recordings). The percent cumulative time spent in seizure decreased by greater than $80 \%$. No changes were made to the patient's antiepileptic drug regimen (topiramate, $72 \mathrm{mg}$ twice a day), and serum drug levels of topiramate remained stable throughout treatment (10 to 12 $\mu \mathrm{g}$ per milliliter at days 13, 68, 209, and 301).

\section{DISCUSSION}

Over the course of treatment to date, milasen appears to have had an acceptable side-effect profile, with no safety concerns. These findings are consistent with those of previous clinical studies of intrathecal delivery of an FDA-approved antisense oligonucleotide for spinal muscular atrophy and investigational antisense oligonucleotides for amyotrophic lateral sclerosis and Huntington's disease, which had similarly acceptable side-effect profiles. ${ }^{8-10,21-23}$ Treatment has also been accompanied by objective reductions in the frequency and duration of seizures.

Rapid development of milasen in this case was possible in large part because of the urgency of the patient's clinical situation, the fact that antisense oligonucleotide drugs can be customized in a sequence-specific fashion, ${ }^{24}$ the precedent of nusinersen (with the same backbone, sugar, and base chemistries) having been safely administered to infants with spinal muscular atrophy, ${ }^{24}$ the favorable uptake and long half-life of this class of drugs in the CNS, ${ }^{13,25}$ and the relative simplicity of manufacturing oligonucleotide drugs (as compared with small molecules or biologics), allowing for timely small-batch manufacturing.

Milasen itself remains an investigational drug, and it is not suited to the treatment of other patients with Batten's disease because its design is customized to our patient's specific mutation. Nonetheless, this experience indicates that antisense oligonucleotides may deserve consideration as a platform for the rapid delivery of individualized treatments. Further exploration of this approach will continue to require careful case-bycase consideration of a number of scientific, clinical, and ethical issues (Table S6), and expectations need to be tempered by the fact that this approach bears substantial risks. At this time, it should be contemplated only in the context of exceptionally serious or life-threatening circumstances. It should also be recognized that only a minority of patients are likely to have mutations that are amenable to the "mRNA splice-switching" strategy deployed here. More generally, this approach is at present probably scalable to only a limited number of patients, given current limitations on systemic and infrastructural issues (e.g., regulatory burden, manufacturing capacity, cost, and reimbursement).

This study illustrates the ability to rationally design, test, and deploy a novel therapeutic agent for a patient with a rare disease on the basis of an understanding of her specific pathogenic mutation. It is an example of individualized genomic medicine. 
Supported by Mila's Miracle Foundation, Boston Children's Hospital, the Harvard Catalyst Clinical and Translational Research Center (through National Center for Advancing Translational Sciences grant 8UL1TR000170), the Mooney Family Fund, and the Boston Children's Hospital Translational Research Program. Claritas Genomics and WuXi NextCODE provided wholegenome sequencing and Clinical Laboratory Improvement Amendments confirmation. The process of obtaining consent, the enrollment of patients, and molecular genetic analyses were conducted with the assistance and support of the Manton Center for Orphan Disease Research and the Boston Children's Hospital Intellectual and Developmental Disabilities Research Center (IDDRC) Molecular Genetics Core Laboratory, which is supported in part by a grant from the National Institutes of Health (1U54HD090255).

Disclosure forms provided by the authors are available with the full text of this article at NEJM.org.
We thank the members of our patient's family for their participation, trust, and partnership, and the many people who contributed their time, advice, and expertise to this project, including Firoz Antia, Frank Bennett, Daniel Capaldi, Mary Ellen Cortizas, Alan Crane, Basil Darras, Tony DeFusco, Craig Dobbs, Diana H. Chin, Didem Demirbas Cakici, Cynthia Gubbels, Luc Chouinard, Richard Finkel, Jim Gombold, Jeff Gulcher, Liz Groebel, Damian Ackermann, Scott Henry, Carl Hull, Cameron Jones, Susan Kornetsky, Art Krieg, Ron Lacro, Helen Legakis, Paul McLean, Steve Miklasz, Patrice Milos, Myriam Mirza, Max Moore, Roger O’Rielly, Philip Reilly, Audrey Saumure Di Fruscia, Susan Srivatsa, Jessica Stewart, John Thompson, Lloyd Tilman, Andy Vick, Hong Vu and colleagues in the Division of Gastroenterology and Inborn Errors Products from the Center for Drug Evaluation and Research at the Food and Drug Administration, Josh Whisenand, David Williams, Fran Wincott, and Michael Wourms.

\section{APPENDIX}

The authors' full names and academic degrees are as follows: Jinkuk Kim, Ph.D., Chunguang Hu, M.D., Ph.D., Christelle Moufawad El Achkar, M.D., Lauren E. Black, Ph.D., Julie Douville, Ph.D., Austin Larson, M.D., Mary K. Pendergast, J.D., Sara F. Goldkind, M.D., Eunjung A. Lee, Ph.D., Ashley Kuniholm, B.S., Aubrie Soucy, B.A., Jai Vaze, B.A., Nandkishore R. Belur, M.S., Kristina Fredriksen, B.S., Iva Stojkovska, B.S., Alla Tsytsykova, Ph.D., Myriam Armant, Ph.D., Renata L. DiDonato, B.S., Jaejoon Choi, Ph.D., Laura Cornelissen, Ph.D., Luis M. Pereira, Ph.D., Erika F. Augustine, M.D., Casie A. Genetti, M.S., Kira Dies, Sc.M., Brenda Barton, R.N., Lucinda Williams, D.N.P., Benjamin D. Goodlett, Ph.D., Bobbie L. Riley, M.D., Amy Pasternak, D.P.T., P.C.S., Emily R. Berry, D.P.T., Kelly A. Pflock, D.P.T., P.C.S., Stephen Chu, Pharm.D., Chantal Reed, Ph.D., Kimberly Tyndall, B.A., Pankaj B. Agrawal, M.B., B.S. M.M.Sc., Alan H. Beggs, Ph.D., P. Ellen Grant, M.D., David K. Urion, M.D., Richard O. Snyder, Ph.D., Susan E. Waisbren, Ph.D., Annapurna Poduri, M.D., M.P.H., Peter J. Park, Ph.D., Al Patterson, Pharm.D., Alessandra Biffi, M.D., Joseph R. Mazzulli, Ph.D., Olaf Bodamer, M.D., Ph.D., Charles B. Berde, M.D., Ph.D., and Timothy W. Yu, M.D., Ph.D.

The authors' affiliations are as follows: the Divisions of Genetics and Genomics (J.K., C.H., E.A.L., A.S., J.V., R.L.D., J.C., P.B.A., A.H.B., S.E.W., O.B., T.W.Y.), Newborn Medicine (P.B.A., P.E.G.), and Neuroradiology (P.E.G.), the Departments of Neurology (C.M.E.A., D.K.U., A. Poduri), Anesthesiology, Critical Care and Pain Medicine (L.C., C.B.B.), Physical and Occupational Therapy (A. Pasternak, E.R.B., K.A.P.), and Pharmacy (S.C., A. Patterson), the Institutional Centers for Clinical and Translational Research (A.K., B.B., L.W.), and the Manton Center for Orphan Disease Research (C.A.G., P.B.A., A.H.B.), Boston Children's Hospital (A.K., A.T., M.A., L.M.P., K.D., B.B., L.W., B.D.G., B.L.R., A.B.), the Department of Biomedical Informatics (J.K., P.J.P.), Harvard Medical School (J.K., C.M.E.A., E.A.L., L.C., B.D.G., B.L.R., P.B.A., A.H.B., P.E.G., D.K.U., S.E.W., P.J.P., A. Patterson, A.B., O.B., C.B.B., T.W.Y.), and the Gene Therapy Program (A.B.), Boston Children's and Dana-Farber Cancer and Blood Disorders Center (A.K., B.B., L.W.), Boston, Charles River Laboratories, Wilmington (L.E.B.), and Broad Institute of MIT and Harvard (E.A.L., O.B., T.W.Y.), Cambridge - all in Massachusetts; Charles River Laboratories, Montreal (J.D.); University of Colorado School of Medicine, Aurora (A.L.); Pendergast Consulting, Washington, DC (M.K.P.); Goldkind Consulting, Potomac, MD (S.F.G.); the Department of Neurology Feinberg School of Medicine, Northwestern University, Chicago (N.R.B., K.F., I.S., J.R.M.); the Department of Neurology, University of Rochester Medical Center, Rochester, NY (E.F.A.); Brain Hz Consulting, Del Mar, CA (C.R.); Tyndall Consulting, Wake Forest, NC (K.T.); and Brammer Bio, Alachua, FL (R.O.S.).

\section{REFERENCES}

1. Griggs RC, Batshaw M, Dunkle M, et al. Clinical research for rare disease: opportunities, challenges, and solutions. Mol Genet Metab 2009;96:20-6.

2. Mole SE, Cotman SL. Genetics of the neuronal ceroid lipofuscinoses (Batten disease). Biochim Biophys Acta 2015; 1852:2237-41.

3. Radke J, Stenzel W, Goebel HH. Human NCL neuropathology. Biochim Biophys Acta 2015;1852:2262-6.

4. Ray DA, Batzer MA. Reading TE leaves: new approaches to the identification of transposable element insertions. Genome Res 2011;21:813-20.

5. Lee E, Iskow R, Yang L, et al. Landscape of somatic retrotransposition in human cancers. Science 2012;337:96771.

6. Wang J, Song L, Grover D, Azrak S,
Batzer MA, Liang P. dbRIP: a highly integrated database of retrotransposon insertion polymorphisms in humans. Hum Mutat 2006;27:323-9.

7. Hancks DC, Ewing AD, Chen JE, Tokunaga K, Kazazian HH Jr. Exon-trapping mediated by the human retrotransposon SVA. Genome Res 2009;19:198391.

8. Finkel RS, Chiriboga CA, Vajsar J, et al. Treatment of infantile-onset spinal muscular atrophy with nusinersen: a phase 2 , open-label, dose-escalation study. Lancet 2016;388:3017-26

9. Finkel RS, Mercuri E, Darras BT, et al. Nusinersen versus sham control in infantile-onset spinal muscular atrophy. $\mathrm{N}$ Engl J Med 2017;377:1723-32.

10. Mercuri E, Darras BT, Chiriboga CA, et al. Nusinersen versus sham control in later-onset spinal muscular atrophy. $\mathrm{N}$ Engl J Med 2018;378:625-35.

11. Singh NK, Singh NN, Androphy EJ, Singh RN. Splicing of a critical exon of human Survival Motor Neuron is regulated by a unique silencer element located in the last intron. Mol Cell Biol 2006;26: 1333-46.

12. Hua Y, Vickers TA, Okunola HL, Bennett CF, Krainer AR. Antisense masking of an hnRNP A1/A2 intronic splicing silencer corrects SMN2 splicing in transgenic mice. Am J Hum Genet 2008;82: 834-48.

13. Rigo F, Chun SJ, Norris DA, et al. Pharmacology of a central nervous system delivered 2'-O-methoxyethyl-modified survival of motor neuron splicing oligonucleotide in mice and nonhuman primates. J Pharmacol Exp Ther 2014;350:46-55. 
14. Smith PJ, Zhang C, Wang J, Chew SL, Zhang MQ, Krainer AR. An increased specificity score matrix for the prediction of SF2/ASF-specific exonic splicing enhancers. Hum Mol Genet 2006;15:2490-508.

15. Fairbrother WG, Yeo GW, Yeh R, et al. RESCUE-ESE identifies candidate exonic splicing enhancers in vertebrate exons. Nucleic Acids Res 2004;32:W187-W190.

16. Chin JJ, Behnam B, Davids M, et al. Novel mutations in CLN6 cause late-infantile neuronal ceroid lipofuscinosis without visual impairment in two unrelated patients. Mol Genet Metab 2019;126:18895.

17. Platt FM, Boland B, van der Spoel AC. The cell biology of disease: lysosomal storage disorders: the cellular impact of lysosomal dysfunction. J Cell Biol 2012; 199:723-34.
18. Brandenstein L, Schweizer M, Sedlacik J, Fiehler J, Storch S. Lysosomal dysfunction and impaired autophagy in a novel mouse model deficient for the lysosomal membrane protein Cln7. Hum Mol Genet 2016;25:777-91.

19. Kousi M, Siintola E, Dvorakova L, et al Mutations in CLN7/MFSD8 are a common cause of variant late-infantile neuronal ceroid lipofuscinosis. Brain 2009;132 810-9.

20. Mitchell NL, Russell KN, Wellby MP, et al. Longitudinal in vivo monitoring of the CNS demonstrates the efficacy of gene therapy in a sheep model of CLN5 Batten disease. Mol Ther 2018;26:2366-78.

21. Miller TM, Pestronk A, David W, et al An antisense oligonucleotide against SOD1 delivered intrathecally for patients with SOD1 familial amyotrophic lateral scle- rosis: a phase 1 , randomised, first-in-man study. Lancet Neurol 2013;12:435-42.

22. Finkel RS, Farwell W. Therapy for spinal muscular atrophy. N Engl J Med 2018; 378:487-8.

23. van Roon-Mom WMC, Roos RAC, de Bot ST. Dose-dependent lowering of $\mathrm{mu}-$ tant huntingtin using antisense oligonucleotides in Huntington disease patients. Nucleic Acid Ther 2018;28:59-62.

24. Hagedorn PH, Yakimov V, Ottosen S, et al. Hepatotoxic potential of therapeutic oligonucleotides can be predicted from their sequence and modification pattern. Nucleic Acid Ther 2013;23:302-10.

25. Geary RS, Norris D, Yu R, Bennett CF. Pharmacokinetics, biodistribution and cell uptake of antisense oligonucleotides. Adv Drug Deliv Rev 2015;87:46-51.

Copyright $(2019$ Massachusetts Medical Society.

JOURNAL ARCHIVE AT NEJM.ORG

Every article published by the Journal is now available at NEJM.org, beginning with the first article published in January 1812. The entire archive is fully searchable, and browsing of titles and tables of contents is easy and available to all.

Individual subscribers are entitled to free 24-hour access to 50 archive articles per year. Access to content in the archive is available on a per-article basis and is also being provided through many institutional subscriptions. 